\title{
Osteomyelitis and septicemia secondary to listeria monocytogenes in a young dog
}

\begin{abstract}
Case description: A young, sexually-intact, male dog was referred for approximately three months duration proliferative, periosteal reaction of multiple bones.

Clinical findings: Culture and sensitivity of both bone and blood grew Listeria monocytogenes. Histopathological examination of bone did not find any evidence of neoplasia.

Treatment and outcome: The patient was started on parenteral antibiotic based on culture and sensitivity results as well as opioid therapy and continued on intravenous fluids with other supportive care as need. The patient rapidly improved. At week 6 the periosteal reaction was still evident. At week 19 the periosteal reaction in all bones had resolved.

Clinical relevance: This case highlights listeriosis as a possible cause of osteomyelitis in dogs. Listeria monocytogenes is a ubiquitous organism within the environment. Certain predisposing factors contribute to the likelihood of developing listeriosis. In our patient, it is likely that there is a deficiency of cell-mediated immunity which allowed clinical disease to occur.
\end{abstract}

Volume 6 Issue I - 2017

Jason Doolittle, Laura Rasmussen
VCA Fort Worth Animal Medical Center, USA

Correspondence: Jason Doolittle,VCA Fort Worth Animal Medical Center, 833I W Freeway Fort Worth, TX 76087, USA Tel 612578509|,Email Doolittle.jason@gmail.com

Received: August 24, 2017 | Published: October 30, 2017

\section{Introduction}

A previously healthy, two-year-old, male (intact) Labrador retriever weighing $25 \mathrm{~kg}$ (55lb) presented with a three-month history of an acute onset of right rear limb and right forelimb lameness. Radiographs obtained during the first examination with his primary care veterinarian revealed a proliferative lesion in the proximal femur at the level of the greater trochanter. A biopsy was obtained at that time. Histopathologic evaluation of this lesion was inconclusive, noting neutrophilic inflammation but no underlying cause or agent was identified. Clindamycin and doxycycline were prescribed, and the patient remained on these medications through to the current presentation.

The patient experienced progressive deterioration in limb function until he was primarily recumbent, developed severe pain in all limbs, and developed a persistent fever. He remained alert with a good appetite and normal urination and defecation. At presentation, the patient was laterally recumbent and exhibited pronounced resistance when attempts were made to assist him to rise. Initial physical exam revealed fever $\left(40^{\circ} \mathrm{C} / 104.0^{\circ} \mathrm{F}\right)$, panting, slight tachycardia (heart rate of 120 beats $/ \mathrm{min}$ ), normal lung sounds and a thin body condition (BCS 3/9). The patient exhibited a marked pain response to palpation of several firm, swellings associated with the right proximal femur, right proximal tibia, right distal radius/proximal carpus, right distal humerus and left distal tibia. There was severe atrophy of the right forequarter and moderate atrophy of the right thigh. No abdominal masses or pain were appreciated. Testicles were both descended and normal on palpation; there was a small amount of purulent preputial discharge. Neurologic exam revealed normal cranial nerves, no pain on spinal palpation, normal limb withdrawal and spinal reflexes in all limbs, spontaneous limb movement and tail wagging, and normal anal tone.
Radiographs revealed polyostotic, proliferative, periosteal reactions with small amounts of lysis and significant soft tissue swelling associated with the proximal/lateral right femur, proximal/ medial right tibia, distal left tibia, distal right humerus and proximal/ medial second right metacarpal bone. A mild, non-structured interstitial pattern was noted in the lung fields. None of the lesions were present on both sides of joints or were associated with joint effusion.

A complete blood count revealed a low red blood cell count $(5.19 \mathrm{M} / \mu \mathrm{l}$; reference range, $5.5-8.5 \mathrm{M} / \mu \mathrm{L})$, a high mean corpuscular volume $(81.3 \mathrm{fL}$; reference range, $60-77 \mathrm{fL})$, and a normal white blood cell count $(10.23 \mathrm{~K} / \mu \mathrm{L}$; reference range, $5.5-16.9 \mathrm{~K} / \mu \mathrm{L})$. Serum biochemical analysis revealed hyperglobulinemia (total globulin concentration, $4.9 \mathrm{~g} / \mathrm{dL}$; reference range, $2.5-4.5 \mathrm{~g} / \mathrm{dL}$ ). All other values for the complete blood count and serum biochemical analysis were within reference ranges. Bone samples from the lesion in the proximal left tibia were collected for histopathology and aerobic/ anaerobic cultures. Blood samples were collected for fungal serology. Pending these results, oral pain medications were administered (carprofen, $2.2 \mathrm{mg} / \mathrm{kg}$ [1mg/lb], PO, q $12 \mathrm{~h}$; tramadol $4 \mathrm{mg} / \mathrm{kg}[1.8 \mathrm{mg} /$ $\mathrm{lb}$ ], PO, q $8 \mathrm{~h})$, a transdermal fentanyl patch was applied $(75 \mu \mathrm{g} / \mathrm{h})$, antibiotics were discontinued, and blood cultures were collected. The patient continued to eat and drink spontaneously.

The patient was afebrile within twelve hours, but over the next 48hours developed progressive, severe neck pain and rigidity, became profoundly reactive and vocal with any touch or manipulation, and was effectively laterally recumbent. Intravenous fluids were initiated (lactated ringers solution, $3 \mathrm{ml} / \mathrm{kg} / \mathrm{hr}[1.4 \mathrm{ml} / \mathrm{lb} / \mathrm{hr}]$, IV), a parenteral opioid was administered as needed for pain (hydromorphone $0.05 \mathrm{mg}$ / $\mathrm{kg}[0.002 \mathrm{mg} / \mathrm{lb}], \mathrm{SQ}, \mathrm{PRN})$, enrofloxacin was administered $(15 \mathrm{mg} / \mathrm{kg}$ $[6.8 \mathrm{mg} / \mathrm{lb}], \mathrm{IV}$, once) and carprofen was discontinued in anticipation of possible corticosteroid use. His appetite remained active, and he was alert and responsive. 
Fungal serology was negative for histoplasma, blastomyces, aspergillus and coccidiodes. Histopathology revealed marked new bone formation with chronic, active inflammation and fibrosis; Gram stain was negative. Anaerobic bone cultures were negative. Aerobic bone cultures on day two revealed a light growth of a single, Gram positive pathogen; further bacteriological identification revealed Listeria monocytogenes. Sensitivity testing revealed the organism to be sensitive to amikacin, amoxicillin, ampicillin, cefadroxil, cefazolin, cephalexin, Clavamox, doxycycline, potentiated sulfonamides, tetracycline; and resistant or intermediate sensitivity to cefpodoxime, clindamycin, difloxacin, enrofloxacin, marbofloxacin. Blood cultures revealed no anaerobic growth, but a positive daytwo growth of Listeria monocytogenes. Sensitivity testing revealed the organism to have the same profile as seen with the organism cultured from the bone samples. Upon receipt of culture results, the patient was started on ampicillin sodium $(10 \mathrm{mg} / \mathrm{kg}[4.5 \mathrm{mg} / \mathrm{lb}]$, $\mathrm{IV}$, q $8 \mathrm{~h})$ and gentamicin sulfate $(6 \mathrm{mg} / \mathrm{kg}[2.7 \mathrm{mg} / \mathrm{lb}], \mathrm{IV}, \mathrm{q} 24 \mathrm{~h})$. Within twelve hours, the patient was moving his neck freely, was able to maintain himself in a sternal recumbent position, and was not painful to general manipulations. Urine concentration and urine sediment was evaluated daily for evidence of concentrating ability and casts. Ampicillin sodium was continued for four days and then discontinued; amoxicillin/clavulanic was thereafter administered orally $(12 \mathrm{mg} / \mathrm{kg}$ [5.5mg/lb], amoxicillin fraction, q $12 \mathrm{~h})$ for 12 weeks. Gentamicin sulfate was continued for seven days, thereafter amikacin sulfate was substituted $(15 \mathrm{mg} / \mathrm{kg}$ [6.8mg/lb], SQ, q $24 \mathrm{~h})$ for two days. After nine days of aminoglycoside administration, hyaline casts were noted in the patient's urine sediment; amikacin was discontinued and doxycycline was initiated $(6 \mathrm{mg} / \mathrm{kg}[2.7 \mathrm{mg} / \mathrm{lb}], \mathrm{PO}, \mathrm{q} 12 \mathrm{~h})$ for 12 weeks.

The patient was ambulatory without assistance within four days of diagnosis and initiation of appropriate therapy. Lameness and bone pain was markedly reduced within eight days. Radiographically evident bone lesions remained on the six-week follow-up films, but these had resolved by the 19 week follow-up. Blood samples were collected the week of diagnosis for immune function testing. An immunoglobulin profile revealed elevations in all fractions (IgG (total) $=6168 \mathrm{mg} / \mathrm{dL}$; reference range, $1000-2000 \mathrm{mg} / \mathrm{dL} ; \mathrm{IgA}=466 \mathrm{mg} /$ $\mathrm{dL}$; reference range, $40-160 \mathrm{mg} / \mathrm{dL} ; \mathrm{IgM}=979 \mathrm{mg} / \mathrm{dL}$; reference range, $100-200 \mathrm{mg} / \mathrm{dL}$ ). Neutrophil function testing revealed low phagocytic activity (38\%; reference range, 50-99\%) and a low oxidative burst response to both phorbol 12-myristate 13-acetate and E. coli ( $\mathrm{PMA}=22 \%$; reference range, $60-93 \%$; E. coli $=24 \%$; reference range, $30-95 \%$ ). The laboratory noted that the test was considered valid for up to 24 hours, but some loss of activity may have occurred due to overnight shipping and handling. Immunophenotyping results were consistent with a reactive lymphocyte population (38\% CD5+, 33\% CD21+, 29\% CD $4+$ and 8\% CD $8+$ cells).

Subsequent immune function testing performed following full clinical recovery and with a clinically normal dog as a control revealed an immunoglobulin profile with substantial elevation in IgG $(7000 \mathrm{mg} / \mathrm{dL}$; reference range $670-1650 \mathrm{mg} / \mathrm{dL})$ and small elevations in $\operatorname{IgA}(286 \mathrm{mg} / \mathrm{dL}$; reference range, $35-270 \mathrm{mg} / \mathrm{dL})$ and $\operatorname{IgM}(548$ $\mathrm{mg} / \mathrm{dL}$; reference range, $100-400 \mathrm{mg} / \mathrm{dL}$ ). Neutrophil function testing revealed continued low phagocytic activity ( $5 \%$; reference range, 50 $99 \%$ ) and low oxidative burst response to both phorbol 12-myristate 13-acetate and E. Coli (PMA=21\%; reference range, 60-93\%; E. Coli $=25 \%$; reference range, $30-95 \%$ ). These values were compared to a control sample handled identically. Immunophenotyping results were consistent with a reactive lymphocyte population (48\% CD5+, $39 \% \mathrm{CD} 21+, 36 \% \mathrm{CD} 4+$ and $37 \% \mathrm{CD} 8+$ cells).

\section{Discussion}

Listeria monocytogenes is a pathogenic, beta-hemolytic, gram-positive, rod-shaped facultative anaerobic bacteria, ${ }^{1}$ and is considered to be ubiquitous in the environment. As a saprophyte, it is commonly isolated from soil, water, sewage, dust, and decaying vegetation. It is commonly found in farm animal feed and silage, and can be found in products made from farm animals. In humans, the main sources of infection tend to be contaminated raw food that is subjected to minimal further processing, such as soft cheeses, frankfurters, pâtés, vegetables and post-processed contaminated milk products. ${ }^{2}$ Humans with impaired cell-mediated immunity are most likely to develop serious clinical symptoms, for example neonates, pregnant women, elderly persons, and immunocompromised patients following transplantation events. ${ }^{3}$ Presumably the predisposing risk factors are similar in animals. Clinical signs are caused by intestinal inflammation and embolic dissemination of the bacteria to distant organs and tissues. Most infections in immunocompetent humans result in acute gastroenteritis or no clinical symptoms. ${ }^{4}$ However, the organism seems to have a predilection for localizing in the CNS and placenta in dogs and cats. ${ }^{1}$

Listeriosis case reports are more extensively written for humans than animals. ${ }^{5-7}$ There are isolated case reports of dogs contracting listeriosis, ${ }^{8-10}$ however none of the cases involve osteomyelitis. Osteomyelitis caused by listeriosis has been reported in several human cases. ${ }^{11,12}$ The primary route of infection is thought to be oral ingestion, and though the incidence is low, the mortality rate in immunocompromised humans is approximately 30\% making $L$. Monocytogenes one of the most deadly human food-borne pathogens, ${ }^{3}$ as seen in the most recent human outbreak involving cantaloupe that resulted in 30 deaths out of 148 confirmed cases. ${ }^{\mathrm{S}}$ As previously noted, this is a ubiquitous pathogen. The relative infrequency with which cases are reported is likely due to the fact that in immunocompetent humans and animals the clinical signs are either mild, non-specific or absent. Consequently, many of those infected likely do not seek treatment.

L. Monocytogenes encounters a range of different environments within the gastrointestinal tract. Such changes include differing levels of acidity, osmolarity, oxygen tension, and bile. Upon encountering the gastrointestinal mucosal epithelium, the first step of the organism is adherence and then invasion of the cells lining the lumen of the intestinal tract. In humans (and certain other mammalian species such as the guinea pig), this is known to occur by direct invasion of the enterocytes. The correct isoform of the receptor molecules, termed internalins, provides the necessary recognition to the Listeria invasion molecules. ${ }^{14}$ The second mechanism of invasion is translocation across the M-cells of Peyer's patches. ${ }^{15}$ This occurs in all species that do not express susceptible isoforms of the receptor molecule. Translocation across M-cells seems to be less efficient than direct invasion of enterocytes. ${ }^{16}$ While many bacteria produce mucinases to allow them to penetrate the mucous layer of the epithelium and come in contact with the cells, this is not the case with Listeria. L. Monocytogenes instead produces a number of surface proteins (internalins) which bind to a specific type of human mucin. ${ }^{17}$ Many bacteria use their flagella not only for motility but also as adhesins or a secretion apparatus. This is also not the case with L. Monocytogenes. 
Instead, L. Monocytogenes only uses flagella for motility, increasing the efficacy of host invasion. . $^{18,19}$

The interaction of internalins with human mucin, Muc2, does not seem to be sufficient for adherence, and further protein expression is necessary. Listeria adhesion protein (LAP) has been shown to bind to host cell heat-shock protein $60,{ }^{20}$ and a fibronectin-binding protein (FbpA) of L. Monocytogenes has been identified to interact with cell surface fibronectin in the murine model..$^{21}$ Internalin A (InlA) promotes the organism's ability to invade the enterocytes. InlB seems to have no direct role in this. Instead, InlB mediates the invasion of hepatocytes and is required for the infection of the fetoplacental unit. ${ }^{22,23}$ InlA binds to E-cadherin. ${ }^{24}$ This interaction is species-specific and is shown to rely on a single amino acid residue in the E-cadherin molecule. ${ }^{16}$ Upon invasion of the cell, L. Monocytogenes appears to become surrounded by the membranes of the phagocytic vacuole. Phospholipases (PI$\mathrm{PlcA}$ and $\mathrm{PI}-\mathrm{PlcB})$ are activated by a metalloprotease (Mpl). These cooperate with the pore-forming hemolysin listeriolysin O (LLO) to accomplish lysis of the phagosome membrane..$^{25,26}$ Once in the cytoplasm, the bacteria begin replicating and make use of specific transporter systems to gain carbohydrates from the host cell. ${ }^{27}$

At the same time of escape from the phagosome, the pathogen induces expression of ActA, a protein that triggers the nucleation and polymerization of host globular g-actin and f-actin filaments. The pattern of polymerization is polarized and leads to a propulsive force that propels the bacteria through the cytoplasm and occasionally into the cytoplasm of neighboring cells. These pseudopods, or so-called "listeriapods", are endocytosed by the adjacent cells. This allows the cell-to-cell spread of $L$. Monocytogenes from one infected cell to another. The bacteria now trapped within the phagosome of the newly infected cell make use of phospholipases and LLO to begin the process anew. This invasion mechanism allows L. Monocytogenes to spread through host tissues without ever leaving the cytosol and not exposing itself to the host adaptive immune response. This fascinating system has been thoroughly reviewed elsewhere. ${ }^{14,27-29}$

Innate immune responses are triggered rapidly following infection of $L$. Monocytogenes and are considered to be essential for survival of the host..$^{30}$ Early resistance to infection is thought to be due to production of interferon- $\gamma(\mathrm{IFN}-\gamma)$ by natural killer cells and the activation of macrophages. ${ }^{31}$ IFN- $\gamma$ and tumor-necrosis factor (TNF) are essential for primary defense against L. Monocytogenes. ${ }^{32,33}$ In experimental models of infection, mice lacking these cytokines or their cognate receptors rapidly die from the infection. ${ }^{34-36}$ Macrophages and neutrophils are believed to be the principal cells responsible for killing Listeria monocytogenes. This has been demonstrated by experiments showing that mice depleted of granulocytes are markedly more susceptible to infection of L. Monocytogenes. ${ }^{37-39}$ While the mechanism for granulocyte or monocyte killing of L. Monocytogenes is not completely understood, the oxidative burst and production of nitrous oxide (NO) both contribute to bacterial clearance in vivo. ${ }^{40}$

Toll-like receptors (TLR) are present on many different immune cell subsets. TLR ligands are present on many different infectious organisms. TLR recognition of pathogen-derived products leads to activation of these cells, which leads to increased production of inflammatory cytokines. Binding of the TLR ligand to its receptor begins a signaling cascade that results in the transcription of NF$\kappa \mathrm{B}$ which results in the expression of different cytokine and antigen presentation related genes. ${ }^{41}$ As an illustration of the important role that TLRs play, mice deficient in MyD88, an important signaling molecule for several TLRs, are highly susceptible to L. Monocytogenes infection. ${ }^{42,43}$ Recent research has found that there is some cellular surveillance system that recognizes infection and responds to it. For example, after escaping the phagosome, live bacteria induce changes in the cell that promote an immune response. Dendritic cells (DCs) that have been infected in vitro with live bacteria express greater levels of co-stimulatory molecules for T-cell activation. Furthermore, they secrete higher levels of pro-inflammatory cytokines than DCs stimulated with heat-killed bacteria. ${ }^{44}$ In macrophages, bacteria that escape the phagosome induce a unique gene expression pattern several hours after infection. ${ }^{45}$ While the exact mechanism of this surveillance system is not known, it is known that it operates independently of TLR signaling. ${ }^{41}$

After a cell recognizes it has become infected, it can attempt to destroy the bacteria through a process called autophagy. ${ }^{46}$ This process involves forming a double membrane around the target (in this case the bacteria, but cells will sometimes recycle organelles through this process). The vacuole then proceeds to the lysosome pathway where the contents are degraded and, in the case of bacteria, destroyed. This process has been very well documented and described for several pathogens including group A Streptococcus and Mycobacterium tuberculosis. ${ }^{47,48}$ Autophagocytosis of $L$. Monocytogenes has only been observed when macrophages are first treated with chloramphenicol. ${ }^{49}$ It is thought that autophagocytosis of L. monocytogenes may be an uncommon occurrence because LLO likely ruptures the vacuole before it reaches the lysosome pathway. DCs play an important role in linking the innate and adaptive immune responses. ${ }^{50}$ TLR recognition of specific bacteria-derived products initiates a signaling cascade that activates the DC. Once activated, the DC expresses costimulatory molecules and cytokines, both of which stimulate T cells. Experimental models have demonstrated that when mice are depleted of DCs, they are unable to generate a CD8 T cell response to infection by L. Monocytogenes ${ }^{51}$ Because L. Monocytogenes resides primarily as an intracellular pathogen, CD4 and CD8 T cells comprise most of the adaptive immune response.

Depending on the type of cell infected, there are several ways in which L. Monocytogenes antigen can be presented to CD8 T cells. The proteins secreted by $L$. Monocytogenes can be degraded by the host proteasome. Once degraded, these peptides are transported to the endoplasmic reticulum where major histocompatibility complex (MHC) class I molecules transport them to the cell surface for recognition by CD $8 \mathrm{~T}$ cells. An alternative pathway is that professional antigen presenting cells (APCs) can present the antigen from bacteria destroyed in lysosomes to CD4 T cells by the MHC class II pathway. It is also known that antigens from the lysosome can traffic to the endoplasmic reticulum where they may be presented to MHC class I molecules. This is a process called cross-presentation. ${ }^{52-54}$ Uninfected APCs can also present bacterial antigen found in debris from dead cells to both CD4 and CD8 T cells. The antigenic presentation of $L$. Monocytogenes depends on the type of cell infected as well as the way in which the antigen was compartmentalized. T cells respond to L. Monocytogenes in a predictable manner. Beginning 4-5days postinfection, antigen-specific $\mathrm{T}$ cells become detectable. The primary response peaks between days 7-9 post-infection. ${ }^{55}$ Following the initial response, the population of antigen-specific $\mathrm{T}$ cells begins to contract to a smaller population of memory $\mathrm{T}$ cells.

L. Monocytogenes typically causes mild clinical symptoms in humans and, presumably, companion animal species. While the exact 
mechanism for infection remains unknown in our patient, he was most likely exposed by oral ingestion. There was no reported history of signs of gastroenteritis preceding the lameness, but these signs may have been self-limiting and overlooked in our patient. During the initial assessment, he also did not have any of the common predisposing immune deficiency factors; for this reason, basic immune function testing was submitted. The body has several defense mechanisms against L. monocytogenes. While the mucosal barrier is considered important to the prevention of disease, it is likely that many bacteria cross this barrier because oral ingestion is the most common route of infection. Once infected the host immune response can be broken down into cell-mediated (or innate) response and humoral (or adaptive) response. From the results of our immune function screening, our patient most likely had a deficiency of cell-mediated immunity. For reasons previously discussed, the adaptive immune system is not the most effective arm of the three protective mechanisms (mucosal barrier, cell-mediated and adaptive immunity) that the body has to prevent listeriosis. Rather, cell-mediated immunity is the predominant immune mechanism the body relies on to prevent infection.

Our patient's cell-mediated immunity was tested on two separate occasions. In both tests his neutrophil population demonstrated low phagocytic activity and low oxidative burst response. These tests are used as markers for overall activity of the cell-mediated immune system. Diminished response to these tests suggests that our patient was unable to mount an effective cell-mediated response to the invading pathogen. It is worth noting that the fact that these results were demonstrated on two separate tests greatly reduces the likelihood that lab error or mishandling caused the decreased neutrophilic response. His humoral immunity did not seem to be affected given the levels of immunoglobulin in his blood. As previously noted, the innate immune system is critical for survival of the patient with infections of Listeria. Because of our patient's innate immunity status, the bacteria was able to survive and even thrive within his body. Given the positive blood cultures and polyostotic involvement, our patient most likely developed osteomyelitis by embolic abscessation; and while there are no CSF samples documenting it, clinical evidence suggests our patient was suffering concurrently with septic Listeria meningitis acquired similarly.

The overall incidence of antibiotic resistance in L. monocytogenes is low. ${ }^{56}$ Most people infected with listeriosis have been treated with penicillin or ampicillin in conjunction with an aminoglycoside. ${ }^{57}$ The current antibiotic treatment of choice for listeriosis ampicillin and gentamicin..$^{58}$ Listeria has been reported to be susceptible to antibiotics active against Gram-positive bacteria ${ }^{59}$ and tetracycline, erythromycin or chloramphenicol, alone or in combination, have been used to treat Listeria infections. ${ }^{60}$ Our patient may not have been able to clear the infection with the initial empirical choice of antibiotic because of resistance to clindamycin. Doxycycline is a bacteriostatic antibiotic and relies on the immune system to clear the infection unlike other antibiotics that directly kill the bacteria. Because our patient's cellmediated immunity was compromised, doxycycline alone was likely insufficient to overcome the infection. It is noted that despite a fever and positive blood cultures indicating septicemia, the total white blood cell count was not elevated. While no definitive reason can be given, it is possible that the patient's body was no longer responding in an acute phase and, therefore, not producing the increased numbers of immature polymorphonuclear leukocytes when the infection first started. However, it is also reasonable to suspect that our patient was either consuming the leukocytes as quickly as they were being produced or that they were being recruited to bone and CNS.

\section{Conclusion}

In conclusion, L. Monocytogenes is a ubiquitous, pathogenic bacterium that most often affects patients with specific predisposing factors; however, otherwise healthy individuals may become infected as well. While listeriosis in immunocompetent humans, and likely in animals, most often causes either febrile gastroenteritis or no clinical symptoms, it should be listed as a differential diagnosis for other infections such as osteomyelitis, epidural abscessation, and abortion. The most severe cases are ones in which the patient is immunocompromised. As previously noted, the mortality rate can be as high as $30 \%$. Despite the need for more aggressive, parenteral treatment in this advanced, multisytemic case, most cases of listeriosis can be treated with oral antibiotics and out-patient supportive care.

The patient in this case report made a full recovery and was free from illness, active and resumed a normal hunting work schedule for twenty-two months. Approximately two years after his Listeria infection, the patient developed multiple, persistent foot pad lesions. Superficial and deep tissue cultures yielded heavy mixed growth of Actinomyces spp, Staphylococcus pseudointermedius, Staphylococcus epidermidis and Escherichia coli with various antibiotic sensitivities. Two separate histopathologic results were consistent with pyogranulomatous inflammation but no organisms could be identified with special stains. The lesions ultimately responded over a 2-3month period to oral penicillin, doxycycline, anti-inflammatory doses of oral steroids, topical silver sulfadiazine, and chlorhexidine foot soaks. No definitive cause of the foot pad lesions was identified, although immune competence of this patient remains in question given the history of these two unusual infectious processes.

\section{Acknowledgements}

None.

\section{Conflict of interest}

Author declares that there is no conflict of interest.

\section{References}

1. Greene CE. Listeriosis: Streptococcal and Other Gram-Positive Bacterial Infections. In: Greene CE, editor. Infectious Diseases of the Dog and Cat. 3rd ed. St Louis: Elsevier; 2006;311-312.

2. Swaminathan B, Gerner-Smidt P. The epidemiology of human listeriosis. Microbes Infect. 2007;9(10):1236-1243.

3. Schuppler M, Loessner MJ. The opportunistic pathogen Listeria monocytogenes: Pathogenicity and interaction with the mucosal immune system. Int J Inflam. 2010;2010:704321.

4. Centers for Disease Control and Prevention. Listeriosis (Listeria infection). 2012.

5. Centers for Disease Control and Prevention. Multistate outbreak of listeriosis associated with Jensen Farms cantaloupe. United States, August--September 2011. MMWR Morb Mortal Wkly Rep. 2011;60(39):1357-1358.

6. Jackson KA, Biggerstaff M, Tobin-D’Angelo M, et al. Multistate outbreak of Listeria monocytogenes associated with Mexican-style cheese made from pasteurized milk among pregnant, Hispanic women. J Food Port. 2011;74(6):949-953.

7. Centers for Disease Control and Prevention. Multistate outbreak of listeriosis--United States, 1998. MMWR Morb Mortal Wkly Rep. 1998;47(50):1085-1086. 
8. Schroder H, van Rensburg IB. Generalised Listeria monocytogenes infection in a dog. J S Afr Vet Assoc. 1993;64(3):133-136.

9. Aoyagi T, Sato Y, Matsuura S, et al. Listeriosis in a raccoon dog (Nyctereutes procyonoides) associated with canine distemper. $J$ Vet Med Sci. 2000;62(6):639-641.

10. Laikko T, Baverud V, Danielsson-Tham M-L, et al. Canine tonsillitis associated with Listeria monocytogenes. Vet Rec. 2004;154:732.

11. Khan KM, Pao W, Kendler J. Epidural abscess and vertebral osteomyelitis cause by Listeria monocytogenes: case report and literature review. Scand J Infect Dis. 2001;33(9):714-716.

12. Chirgwin K, Gleich S. Listeria monocytogenes osteomyelitis. Arch Intern Med. 1989;149(4):931-932.

13. Ooi ST, Lorber B. Gastroenteritis due to Listeria monocytogenes. Clin Infect Dis. 2005;40(9):1327-1332.

14. Vázquez-Boland JA, Kuhn M, Berche P, et al. Listeria pathogenesis and molecular virulence determinants. Clin Microbiol Rev. 2001;14(3):584-640.

15. Corr S, Hill C, Gahan CGM. An in vitro cell-culture model demonstrates internalin-and hemolysin-independent translocation of Listeria monocytogenes across M cells. Microb Pathog. 2006;41(6):241-250.

16. Lecuit M. Understanding how Listeria monocytogenes targets and crosses host barriers. Clin Microbiol Infect. 2005;11(6):430-436.

17. Lindén SK, Bierne $\mathrm{H}$, Sabet $\mathrm{C}$, et al. Listeria monocytogenes internalins bind to human intestinal mucin MUC2. Arch Microbiol. 2008;190(1):101-104.

18. Bigot A, Pagniez H, Botton E, et al. Role of FliF and FliL of Listeria monocytogenes in flagellar assembly and pathogenicity. Infect Immun. 2005;73(9):5530-5539.

19. O’Neil HS, Marquis H. Listeria monocytogenes flagella are used for motility, not as adhesins, to increase host cell invasion. Infect Immun. 2006;74(12):6675-6681

20. Wampler JL, Kim K-P, Jaradat Z, et al. Heat shock protein 60 acts as a receptor for Listeria adhesion protein in Caco-2 cells. Infect Immun. 2004;72(2):931-936.

21. Dramsi S, Bourdichon F, Cabanes D, et al. FbpA, a novel multifunctional Listeria monocytogenes virulence factor. Mol Microbiol. 2004;53(2):639-649.

22. Disson O, Grayo S, Huillet E, et al. Conjugated action of two species-specific invasion proteins for fetoplacental listeriosis. Nature. 2008;455(7216):1114-1118.

23. Khelef N, Lecuit , Bierne H, et al. Species specificity of the Listeria monocytogenes InlB protein. Cell Microbiol. 2006;8(3):457-470.

24. Mengaud J, Lecuit M, Lebrun M, et al. Antibodies to the leucine-rich repeat region of internalin block entry of Listeria monocytogenes into cells expressing E-cadherin. Infect Immun. 1996;64(12):5430-5433.

25. Marquis H, Doshi V, Portnoy DA. The broad-range phospholipase C and a metalloprotease mediate listeriolysin O-independent escape of Listeria monocytogenes from a primary vacuole in human epithelial cells. Infect Immun. 1995;63(11):4531-4534.

26. Smith GA, Marquis H, Jones S, et al. The two distinct phospholipase $\mathrm{C}$ of Listeria monocytogenes have overlapping roles in escape from a vacuole and cell-to-cell spread. Infect Immun. 1995;63(11):4231-4237.

27. Kuhn M, Scortti M, Vázquez-Boland JA. Pathogenesis. In: Liu D, editor. Handbook of Listeria monocytogenes. Boca Raton: Taylor \& Francis CRC Press; 2008:97-136.
28. Hamon M, Bierne H, Cossart P. Listeria monocytogenes: a multifaceted model. Nat Rev Microbiol. 2006;4(6):423-434.

29. Dussurget O, Pizarro-Cerda J, Cossart P. Molecular determinants of Listeria monocytogenes virulence. Annu Rev Microbiol. 2004;58:587-610.

30. Unanue ER. Studies in listeriosis show the strong symbiosis between the innate cellular system and the T-cell response. Immunol Rev. 1997;158(1):11-25.

31. Tripp CS, Wolf SF, Unanue ER. Interleukin 12 and tumor necrosis factor $\alpha$ are costimulators of interferon $\gamma$ production by natural killer cells in severe combined immunodeficiency mice with listeriosis, and interleukin 10 is a physiologic antagonist. Proc Natl Acad Sci USA. 1993;90(8):3725-3729.

32. Buchmeier NA, Schreiber RD. Requirement of endogenous interferon- $\gamma$ production for resolution of Listeria monocytogenes infection. Proc Natl Acad Sci USA. 1985;82(21):7404-7408.

33. Havell EA. Evidence that tumor necrosis factor has an important role in antibacterial resistance. J Immunol. 1989;143(9):2894-2899.

34. Pfeffer K, Matsuyama T, Kündig TM, et al. Mice deficient for the $55 \mathrm{kd}$ tumor necrosis factor receptor are resistant to endotoxic shock, yet succumb to L. monocytogenes infection. Cell. 1993;73(3):457-467.

35. Rothe J, Lesslauer W, Lötscher H, et al. Mice lacking the tumour necrosis factor receptor 1 are resistant to IMF-mediated toxicity but highly susceptible to infection by Listeria monocytogenes. 1993;364(6440):798-802.

36. Harty JT, Bevan MJ. Specific immunity to Listeria monocytogenes in the absence of IFN $\gamma$. Immunity. 1995;3(1):109-117.

37. Conlan JW, North RJ. Neutrophils are essential for early anti-Listeria defense in the liver, but not in the spleen or peritoneal cavity, as revealed by granulocyte-depleting monoclonal antibody. $J$ Exp Med. 1994;179(1):259-268.

38. Rogers HW, Unanue ER. Neutrophils are involved in acute, nonspecific resistance to Listeria monocytogenes in mice. Infect Immunol. 1993;61(12):5090-5096.

39. Czuprynski CJ, Brown JF, Maroushek N, et al. Administration of anti-granulocyte mAb RB6-8C5 impairs the resistance of mice to Listeria monocytogenes infection. J Immunol. 1994;152(4):1836-1846.

40. Pamer EG. Immune responses to Listeria monocytogenes. Nat Rev Immunol. 2004;4(10):812-823.

41. Zenewicz LA, Shen H. Innate and adaptive immune responses to Listeria monocytogenes: a short review. Microbes Infect. 2007;9(10):1208-1215.

42. Seki E, Tsutsui H, Tsuji NM, et al. Critical roles of myeloid differentiation factor 88-dependent proinflammatory cytokine release in early phase clearance of Listeria monocytogenes in mice. J Immunol. 2002;169(7):3869-3875.

43. Edelson BT, Unanue ER. MyD88-dependent but Toll-like receptor 2-independent innate immunity to Listeria: no role for either in macrophage listericidal activity. J Immunol. 2002;169(7):3869-3875.

44. Brzoza KL, Rockel AB, Hiltbold EM. Cytoplasmic entry of Listeria monocytogenes enhances dendritic cell maturation and T cell differentiation and function. J Immunol. 2004;173(4):2641-2651.

45. McCaffrey RL, Fawcett P, O'Riordan M, et al. A specific gene expression program triggered by Gram-positive bacteria in the cytosol. Proc Natl Acad Sci USA. 2004;101(31):11386-11391.

46. Deretic V. Autophagy in innate and adaptive immunity. Trends Immunol. 2005;26(10):523-528. 
47. Nakagawa I, Amano A, Mizushima N, et al. Autophagy defends cells against invading group A streptococcus. Science. 2004;306(5698):1037-1040.

48. Gutierrez MG, Master SS, Singh SB, et al. Autophagy is a defense mechanism inhibiting BCG and Mycobacterium tuberculosis survival in infected macrophages. Cell. 2004;119(6):753-766.

49. Rich KA, Burkett C, Webster P. Cytoplasmic bacteria can be targets for autophagy. Cill Microbiol. 2003;5(7):455-468.

50. Medzhitov R. Toll-like receptors and innate immunity. Nat Rev Immunol. 2001;1(2):135-145.

51. Jung S, Unutmaz D, Wong $\mathrm{P}$, et al. In vivo depletion of CD11c $(+)$ dendritic cells abrogates priming of CD $8(+) \mathrm{T}$ cells by exogenous cell-associated antigens. Immunity. 2002;17(2):211-220.

52. Heath WR, Carbone FR (2001) Cross-presentation, dendritic cells, tolerance and immunity. Annu Rev Immunol. 2001;19:47-64.

53. den Haan JM, Bevan MJ. Antigen presentation to CD8+ T cells: cross-priming in infectious diseases. Curr opin Immunol. 2001;13(4):437-441.
54. Guermonprez P, Saveanu L, Kleijmeer M, et al. ER-phagosome fusion defines an MHC class I cross-presentation compartment in dendritic cells. Nature. 2003;425(6956):397-402.

55. Busch DH, Pilip IM, Vijh S, et al. Coordinate regulation of complex $\mathrm{T}$ cell pupluations responding to bacterial infection. Immunity. 1998;8(3):353-362.

56. Walsh D, Duffy G, Sheridan JJ, et al. Antibiotic resistance among Listeria, including Listeria monocytogenes, in retail foods. J Appl Microbiol. 2001;90(4):517-522.

57. Charpentier E, Courvalin P. Antibiotic resistance in Listeria spp. Antimicrob Agents Chemother. 1999;43(9):2103-2108.

58. Lorber B. Listeriosis. Clin Infect Dis. 1997;24(1):1-11.

59. Hawkins AE, Bortolussi R, Issekutz AC. In vitro and in vivo activity of various antibiotics against Listeria monocytogenes type 4b. Clin Invest Med. 1984;7(4):335-341.

60. Hof H. Therapeutic activities of antibiotics in listeriosis. Infection. 1991;19(Suppl 4):229-233. 